\title{
Probing for Chemically Functional Groups on Graphene Oxide in an Aberration- Corrected Electron Microscope
}

\author{
R. Cieslinski, ${ }^{1}$ S. Rozeveld, ${ }^{1}$ J. Huang, ${ }^{2}$ P. Specht,${ }^{3}$ C. Kisielowski, ${ }^{4}$ \\ ${ }^{1}$ Dow Chemical Company, Midland, MI 48642 \\ ${ }^{2}$ Carl Zeiss SMT Inc, Peabody, MA 01960 \\ ${ }^{3}$ University of California, Materials Science and Engineering, Berkeley CA 94720 \\ ${ }^{4}$ National Center for Electron Microscopy, Lawrence Berkeley National Laboratory, One Cyclotron \\ Rd . Berkeley CA 94720
}

The next generation electron microscope is currently being developed within the DoE's TEAM Project [1]. A prototype instrument (TEAM 0.5) became recently operational [2] and provided phase contrast images of graphene - a single sheet of carbon atoms - with truly atomic resolution and extraordinary signal to noise ratios [3]. Conventional electron microscopes do not provide such performance (Figure 1) because unlike other microscopes TEAM 0.5 is equipped with a Cs corrector, a prototype high brightness gun / monochromator assembly and can be operated at $80 \mathrm{kV}$. The ability to directly image the atomic structure of graphene opens the possibility to probe for chemically functional groups such as oxidized rings containing $\mathrm{C}-\mathrm{O}-\mathrm{C}$ (epoxide or ether) or $\mathrm{C}-\mathrm{OH}$ groups. When functionalized, single-sheet graphene is expected to serve in a variety of applications.

Graphene oxide films were fabricated by a modified version of the Staudenmaier process [4]. The chemical composition of the film was determined by X-ray Photoelectron Spectroscopy technique. Flakes of such materials were deposited on holey carbon grids and exposed to the high brightness beam of the electron microscope to free the graphene oxide from adsorbents locally. Focus series of lattice images were recorded from cleaned areas and reconstructed to obtain amplitude and phase of the scattered electron beam using a Gerchberg-Saxton algorithm. Figure 2 shows a reconstructed phase image of a graphene oxide film. Grains of 2-3 nm diameter are seen and single graphene layers as well as double layers are readily observed. In other instances we detected rotational stacking faults [5] in such materials that are significantly strained (Fig.2). Schniepp et al. [6] have attributed the strain and film bending to the presence of epoxide groups. Clearly the identification of such group in the reconstructed phase image requires extensive image simulations as well as an estimate of statistical and systematic errors because image patterns change rapidly across the picture. In this contribution we will provide such data in order to set boundary conditions for the detection of oxygen in graphene.

\section{References}

1. http://ncem.lbl.gov/TEAM-project/index.html

2. C. Kisielowski, B. Freitag, M. Bischoff, H. van Lin, et al., Microscopy and Microanalysis 14 (2008) 454

3. J. Meyer, C. Kisielowski, R. Erni, M. D. Rossell, M. F. Crommie, A. Zettl, Nano Lett., 2008, 8, 3582-3586

4. L. Staudenmaier, Ber. Dtsch. Chem. Ges. (1859), 149, 249.

5. J. H. Warner, M. H. Rümmeli Thomas Gemming, B. Büchner, et al. Nano Lett., 2009, 9, 102-10

6. H. C. Schniepp, Je-Luen Li, M. J. McAllister, et al. J. Phys. Chem. B, DOI: 10.1021/jp060936f, 2006

7. The TEAM project is supported by the Department of Energy, Office of Science, Basic Energy Sciences. 


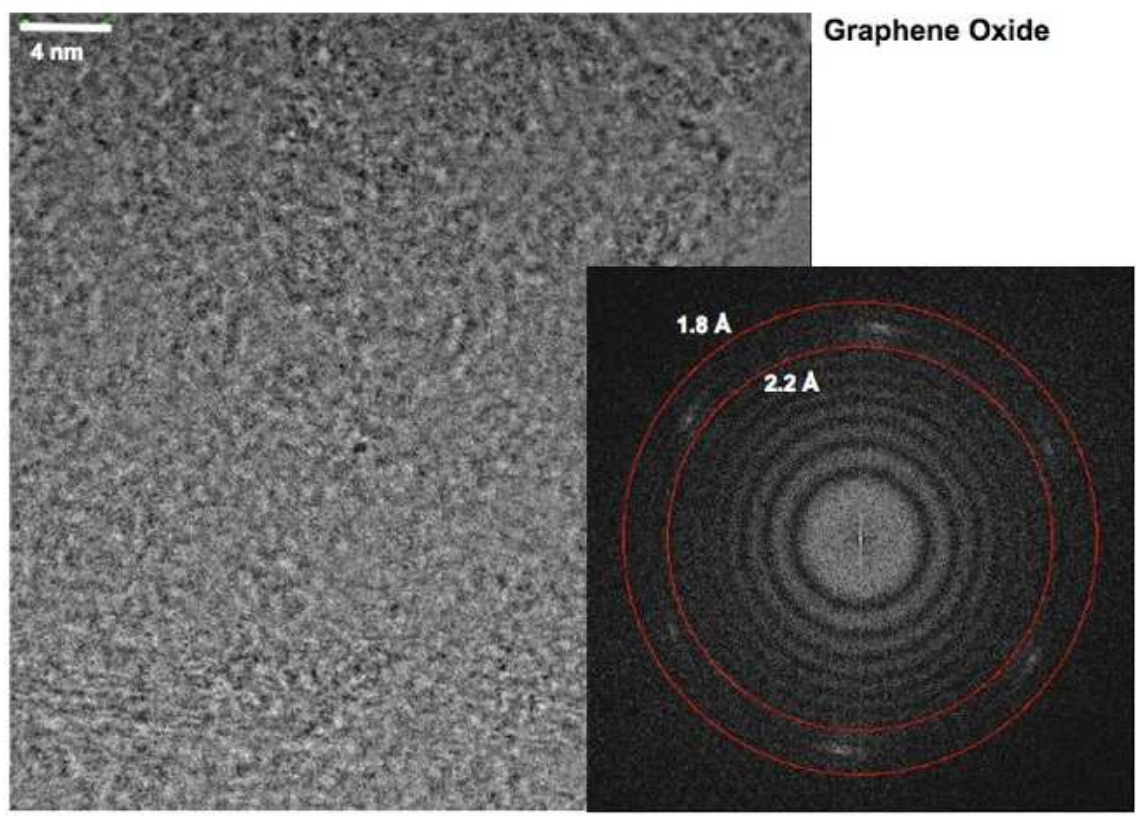

FIG. 1. Lattice image of graphene oxide. $300 \mathrm{kV}$. Image Fourier components (inset) at $2 \AA$ do not allow for atomic resolution. The presence of adsorbents in combination with the finite spherical aberration constant $\mathrm{Cs}=0.6 \mathrm{~mm}$ introduce random intensity fluctuation that obscure the visualization of the lattice pattern.

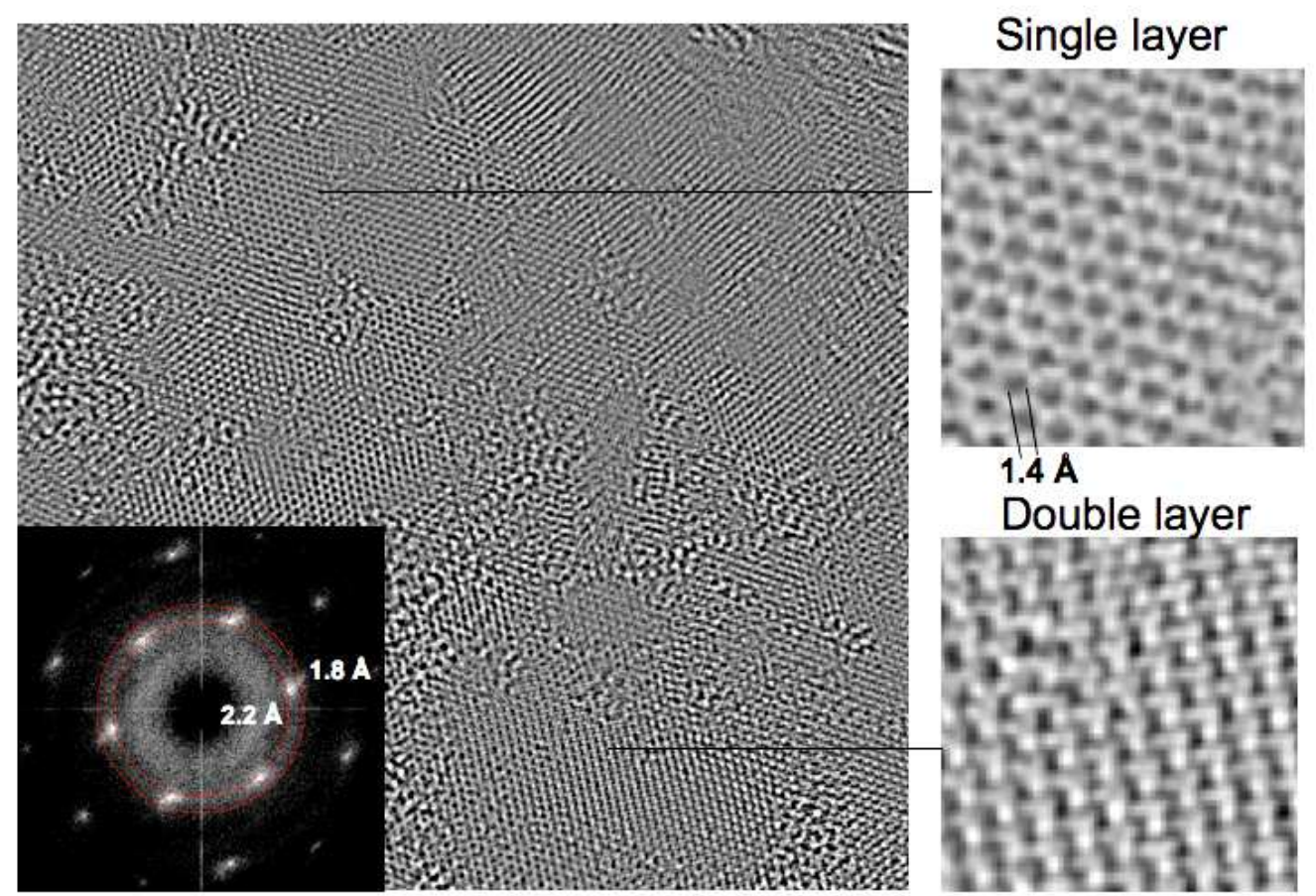

FIG. 2. Reconstructed phase image of graphene oxide. TEAM 0.5, $80 \mathrm{kV}$. Coherently ordered grains of 2-3 nm diameter are visible. The layers are strained as indicated by the directional streaking of the image Fourier components. Single and double layers are readily observed. 